\title{
PANO PRETO IMPREGNADO COM FUNGO Beauveria bassiana REDUZ A SOBREVIVÊNCIA DE FÊMEAS DE Aedes aegypti
}

\author{
Anderson Ribeiro ${ }^{1}$ \\ Adriano Rodrigues de Paula ${ }^{2}$ \\ Leila Eid Imad da Silva ${ }^{3}$ \\ Richard lan Samuels ${ }^{4}$
}

Resumo: O mosquito Aedes aegypti é o principal vetor das doenças dengue, chikungunya e zika. $O$ presente estudo teve o objetivo avaliar a taxa de sobrevivência de fêmeas de A. aegypti infectadas com o fungo entomopatogênico Beauveria bassiana em condições de laboratório. Para infecção os mosquitos foram expostos, por 48 horas, ao pano preto impregnado com B. bassiana pendurado dentro de um pote de plástico e depois a taxa de sobrevivência foi avaliada diariamente por 7 dias. Os mosquitos expostos ao B. bassiana tiveram, no sétimo dia de avaliação, $16 \%$ de sobrevivência, valor significativamente diferente do controle ( $80 \%$ de insetos vivos). O presente estudo mostrou que fêmeas de A. aegypti foram suscetíveis ao fungo B. bassiana. A redução da população de mosquitos A. aegypti diminuirá a transmissão de dengue, chikungunya e zika.

Palavras-chave: Mosquitos; Fungos; Dengue; Zika; Chikungunya.

\footnotetext{
1 Universidade Estadual do Norte Fluminense - Centro de Ciências e Tecnologias Agropecuárias - Laboratório de Entomologia e Fitopatologia, Av. Alberto Lamego, 2000, Campos dos Goytacazes-RJ, Brasil. E-mail: anderson.ribeirorj@yahoo.com.br.

2 Universidade Estadual do Norte Fluminense - Centro de Ciências e Tecnologias Agropecuárias - Laboratório de Entomologia e Fitopatologia, Av. Alberto Lamego, 2000, Campos dos Goytacazes-RJ, Brasil. E-mail: biodepaula@yahoo.com.br.

3 Universidade Estadual do Norte Fluminense - Centro de Ciências e Tecnologias Agropecuárias - Laboratório de Entomologia e Fitopatologia, Av. Alberto Lamego, 2000, Campos dos Goytacazes-RJ, Brasil. E-mail: imad.saudecoletiva@gmail.com.

4 Universidade Estadual do Norte Fluminense - Centro de Ciências e Tecnologias Agropecuárias - Laboratório de Entomologia e Fitopatologia, Av. Alberto Lamego, 2000, Campos dos Goytacazes-RJ, Brasil. E-mail: richard@uenf.br.
} 